\title{
EFICIÊNCIA DE HERBICIDAS NO CONTROLE DE PLANTAS DANINHAS LATIFOLIADAS EM MAMONA
}

\section{H.S. Vitorino, D. Martins, S.Í.A. Costa, R.P. Marques, G.S.F. de Souza, C.F. de Campos}

Universidade Estadual Paulista, Faculdade de Ciências Agrárias, Departamento de Produção Vegetal, Setor de Produção e Melhoramento Vegetal, CP 237, CEP 18603-970 Botucatu, SP, Brasil. E-mail: vitorinohermeson@fca.unesp.br

\section{RESUMO}

\begin{abstract}
O objetivo do presente trabalho foi avaliar a eficiência de diferentes herbicidas, aplicados em jato dirigido, no controle de plantas daninhas dicotiledôneas na cultura da mamona. Os herbicidas testados foram: saflufenacil $\left(28,40\right.$ e $\left.56 \mathrm{~g} \mathrm{ha}^{-1}\right)$, carfentrazone-ethyl $\left(30 \mathrm{~g} \mathrm{ha}^{-1}\right)$, flumioxazin $\left(25 \mathrm{~g} \mathrm{ha}^{-1}\right)$, glyphosate $\left(720 \mathrm{~g} \mathrm{ha}^{-1}\right)$ e glyphosate + saflufenacil $\left(720+28 \mathrm{~g} \mathrm{ha}^{-1}\right)$ aplicados em pós-emergência em três espécies de plantas daninhas (Richardia brasilienses, Sida rhombifolia e Ipomoea grandifolia), além de uma testemunha sem aplicação de herbicida. Os herbicidas foram pulverizados quando as plantas de mamona apresentavam 0,50 a $0,70 \mathrm{~m}$ de altura. As avaliações visuais de controle foram realizadas aos 3, 7, 14, 21 e 28 dias após a aplicação dos tratamentos. O estudo foi instalado no delineamento experimental em blocos casualizados, com três repetições. O herbicida saflufenacil foi eficiente no controle de todas as plantas daninhas estudadas, sendo que os herbicidas carfentrazone-ethyl e flumioxazin obtiveram apenas controles satisfatórios das plantas daninhas. A mistura testada foi eficiente no controle de todas as plantas daninhas.
\end{abstract}

PALAVRAS-CHAVE: Carfentrazone-ethyl, Ricinus communis, flumioxazin, saflufenacil.

\section{ABSTRACT}

EFFECTIVENESS OF HERBICIDES ON WEED CONTROL IN CASTOR BEANS. The objective of this study was to evaluate the effectiveness of different herbicides, applied in direct spray, to control dicotyledonous weeds in castor beans. The herbicides tested were saflufenacil $(28,40$ and $56 \mathrm{~g}$ ha-1), carfentrazone-ethyl $\left(30 \mathrm{~g} \mathrm{ha}^{-1}\right)$, flumioxazin $\left(25 \mathrm{~g} \mathrm{ha}^{-1}\right)$, glyphosate $\left(720 \mathrm{~g} \mathrm{ha}^{-1}\right)$ and glyphosate + saflufenacil $\left(720+28 \mathrm{~g} \mathrm{ha}^{-1}\right)$ applied post-emergence on three species of weeds (Richardia brasiliensis, Sida rhombifolia and Ipomoea grandifolia), and a control without herbicide. The herbicides were sprayed when the castor bean plants showed 50 to $70 \mathrm{~cm}$ of height. Visual assessments of the degree of control were performed at 3, 7, 14, 21 and 28 days after treatment application. The study was installed in a randomized complete block design with three replications. The herbicide saflufenacil was effective in the control of all the weeds studied, and the herbicides carfentrazone-ethyl and flumioxazin presented only satisfactory control of weeds. The mixture tested was effective in the control of all weeds.

KEY WORDS: Carfentrazone-ethyl, Ricinus communis, flumioxazin, saflufenacil.

A mamoneira, conhecida cientificamente como Ricinus communis L, é uma xerófila de origem afro-asiática da família das euforbiáceas, classe dicotiledônea, ordem gerianáceas. É bastante tolerante a escassez de água, porém, exigente em calor e luminosidade. Está disseminada em quase todo o Nordeste, cujas condições climáticas são propícias ao seu desenvolvimento e crescimento, nos locais já zoneados pela Embrapa e referendado pelo Ministério da Agricultura, Pecuária e Abastecimento (EMBRAPA, 2006).

A mamoneira cultivada em espaços mais amplose em menor densidade comousualmenteutilizadas em culturas como feijão, milho e soja pode reduzir a sua habilidade competitiva (MAcIEL et al., 2004) efavorece o crescimento de espécies vegetais oportunistas, as quais competem com a cultura por recursos do meio.

A cultura da mamona, como toda cultura, está sujeita à interferência das plantas daninhas. Muitos herbicidas são utilizados para controle da mamona em outras culturas. Isto implica em dificuldades em encontrar herbicidas que controlem plantas daninhas dicotiledôneas em mamona, e que ao mesmo tempo sejam seletivos a esta cultura.

De fato, o único herbicida legalmente habilitado para ser aplicadona lavoura demamona éo herbicida 
trifluralin, aplicado em pré-emergência (AzEvEDO; SEVERINO, 2006). Uma das maiores dificuldades do controle químico de plantas daninhas em mamona é a falta de registro de herbicidas seletivos a essa cultura, principalmente daqueles aplicados após a emergência e que controlam plantas daninhas dicotiledôneas (folhas-largas). Alguns estudos, contudo, avaliaram a viabilidade de utilização dos herbicidas chlorimuron-ethyl e do halosulfuron (MACIEL et al., 2004). Pela dificuldade de encontrar herbicidas seletivos à mamoneira, o uso de aplicações em jato dirigido consiste em uma forma viável de aplicação de herbicidas para a cultura. Tal forma de aplicação possibilita o uso de herbicidas de ampla ação, como o glyphosate, carfentrazone-ethl, flumioxazin e o saflufenacil, sendo este último de amplo espectro sobre plantas daninhas de folha larga.

O glyphosate é um inibidor da síntese dos aminoácidos aromáticos como tirosina, fenilalanina e triptofano por atuar na enzima precursora EPSPs evitando a transformação do shiquimato em corismato (SHANER; BRIDGES, 2003).

O carfentrazone-ethyl (do grupo químico das Triazolinonas) e o flumioxazin (do grupo químico da $\mathrm{N}$-fenilftalimida) são herbicidas cujo mecanismo de ação está relacionado com a inibição da enzima protoporfirogênio oxidase(PPO ou PROTOX), responsável por uma das etapas de síntese da clorofila, muito utilizado no controle de plantas daninhas aplicados em pós-emergência (DuKE etal.,1991). Já o saflufenacil (do grupo dos Difenil-etéres), depois de aplicado, demonstra o mesmo tipo de sintoma fitotoxico, com rápido murchamento dependente de luz e rápido crescimento da necrose no tecido, com a inibição da PROTOX, que são herbicidas de peroxidação de plantas susceptíveis (Grossmann et al., 2010).

O objetivo do presente trabalho foi avaliar a eficiência de diferentes herbicidas, aplicados em jato dirigido, no controle de plantas daninhas dicotiledôneas na cultura da mamona.

O experimento foi conduzido na Fazenda Experimental Lageado, no Município de Botucatu, SP, pertencente à FCA/UNESP, em um solo classificado como Nitossolo Vermelho Estruturado, com as seguintes características: areia (57\%), silte (18\%), argila ( $25 \%)$ e saturação de bases (57\%).

Os herbicidas testados foram: saflufenacil+Dash $\left(28,40\right.$ e $\left.56 \mathrm{~g} \mathrm{ha}^{-1}+0,5 \mathrm{v} / \mathrm{v}\right)$, carfentrazone-ethyl + assist (30 $\left.\mathrm{g} \mathrm{ha}^{-1}+0,5 \mathrm{v} / \mathrm{v}\right)$, flumioxazin $\left(25 \mathrm{~g} \mathrm{ha}^{-1}\right.$ $+0,5 \mathrm{v} / \mathrm{v})$, glyphosate $\left(720 \mathrm{~g} \mathrm{ha}^{-1}\right)$ e glyphosate + saflufenacil $\left(720+28 \mathrm{~g} \mathrm{ha}^{-1}+0,5 \mathrm{v} / \mathrm{v}\right)$ aplicados em pós-emergência em três espécies de plantas daninhas (Richardia brasilienses Gomes, Sida rhombifolia L. e Ipomoea grandifolia Drammer), além de uma testemunha sem aplicação de herbicida. As plantas daninhas encontravam-se distribuidas na área com 6, 28 e 19 plantas por metro quadrado, para $I$. grandifolia, $R$. brasilienses e $S$. rhombifolia, respectivamente. A aplicação dos herbicidas foi realizada em 4/4/2009, sendo que, no momento da aplicação, as plantas daninhas apresentavam-se com 4 a 6 pares de folhas definitivas. As parcelas foram alocadas na área com as seguintes dimensões: 3,0 m de largura por 5,0 m de comprimento. Os tratamentos químicos foram aplicados utilizando-se um pulverizador costal, pressurizado a $\mathrm{CO}_{2}$, equipado com um reservatório de $2 \mathrm{~L}$. O equipamento foi regulado para proporcional um consumo de calda de $200 \mathrm{~L} \mathrm{ha}^{-1}$. A barra de aplicação constituía-se de duas pontas do tipo jato plano "Teejet" XR 11002 VS, distanciados $50 \mathrm{~cm}$ entre si. A pulverização foi realizada em jato dirigido na entrelinha da cultura. As plantas de mamona apresentavam altura que variava de 0,50 a 0,70 m no momento da aplicação.

As avaliações visuais de controle e fitotoxicidade foram realizadas aos 3, 7, 14, 21 e 28 dias após a aplicação dos tratamentos (DAA), através de uma escala de percentual de notas, na qual 0 (zero) correspondeu a nenhumainjúria demonstrada pelas plantase 100 (cem) a morte das plantas, segundo a Sociedade Brasileira da Ciência das Plantas Daninhas - SBCPD (1995).

Os tratamentos foram dispostos em um delineamento experimental de blocos casualizados com quatro repetições, sendo os resultados submetidos a analise de variância pelo teste " $\mathrm{F}$ " e suas médias comparadas através do Teste Tukey $(p>0,05)$ utilizando o programa estatístico SISVAR 5.0.

Em todo o período experimental não foram observados sintomas de injúrias nas folhas do dossel das plantas de mamona. Leves cloroses ocorreram inicialmente nos caules das plantas com a aplicação de carfentrazone-ethyl, flumioxazin e saflufenacil aplicadoisolado (menoresque $4 \%$ ), quese dissiparam no decorrer do período experimental.

Aos 3 DAA dos herbicidas sobre as plantas de $R$. brasilienses (poaia-branca) observa-se que as duas doses maiores de saflufenacil e de sua mistura com glyphosate proporcionaram controles satisfatórios das plantas daninhas. A menor dose de saflufenacil e os herbicidas flumioxazin e glyphosate aplicados isoladamente ainda apresentavam controles insatisfatórios (Tabela 1).

Registra-se que apenas os herbicidas carfentrazone-ethyl e glyphosate aplicados isoladamente não proporcionaram um controle satisfatório das plantas de poaia-branca aos 7 DAA. Já, aos 14 DAA dos herbicidas, nota-se um excelente controle da planta daninha também com o uso de glyphosate de forma isolada. Ressalta-se que a menor dose de saflufenacil e carfentrazone-ethyl proporcionou controles considerados insatisfatórios das plantas de poaia-branca, o que não corrobora com os dados observado por MONQUEROetal. (2001), que o herbicida carfentrazoneethyl controlou 95\% dessa planta daninha. 
Tabela 1 - Efeito de diferentes tratamentos químicos sobre o controle de Richardia brasilienses na cultura da mamona, em diferentes dias após a aplicação de herbicidas. Botucatu, SP, 2009.

\begin{tabular}{|c|c|c|c|c|c|c|}
\hline \multirow{2}{*}{ Tratamentos } & Doses & \multicolumn{5}{|c|}{ (\%) Controle } \\
\hline & (g i.a./e.a. ha-1) & $3 \mathrm{DAA}^{(3)}$ & $7 \mathrm{DAA}$ & $14 \mathrm{DAA}$ & $21 \mathrm{DAA}$ & 28 DAA \\
\hline saflufenacil $^{(1)}$ & 28 & $66,50 \mathrm{~b}$ & $92,75 a$ & $75,50 \mathrm{ab}$ & $57,75 b c$ & $60,75 b c$ \\
\hline saflufenacil (1) & 40 & $84,00 \mathrm{a}$ & $88,75 a$ & $88,75 \mathrm{ab}$ & $75,00 \mathrm{abc}$ & $84,75 \mathrm{ab}$ \\
\hline saflufenacil $^{(1)}$ & 56 & $79,00 \mathrm{ab}$ & $98,00 \mathrm{a}$ & $91,75^{\mathrm{a}}$ & $78,25 \mathrm{ab}$ & $86,00 \mathrm{ab}$ \\
\hline carfentrazone-ethyl ${ }^{(2)}$ & 25 & $51,50 \mathrm{~b}$ & $31,25 c$ & $63,00 \mathrm{~b}$ & $45,25 c$ & $49,25 c$ \\
\hline flumioxazin ${ }^{(2)}$ & 30 & $68,25 \mathrm{ab}$ & $84,50 \mathrm{ab}$ & $94,25^{\mathrm{a}}$ & $90,25 \mathrm{ab}$ & $92,25 a$ \\
\hline glyphosate & 720 & 0c & $63,75 b$ & $98,50^{\mathrm{a}}$ & $99,00 \mathrm{a}$ & $99,50 \mathrm{a}$ \\
\hline glyphosate+saflufenacil $^{(1)}$ & $720+28$ & $88,00 \mathrm{a}$ & $96,00 \mathrm{a}$ & $97,75^{\mathrm{a}}$ & $91,25^{\mathrm{a}}$ & $96,50 \mathrm{a}$ \\
\hline $\mathrm{F}_{\text {Tratamento }}$ & & $33,69 * *$ & $57,90 * *$ & $34,09 * *$ & $22,02 * *$ & $25,83^{* *}$ \\
\hline $\mathrm{F}_{\text {bloco }}$ & & $7,12^{* *}$ & $3,964^{*}$ & $3,460^{*}$ & $3,17^{*}$ & $4,37^{*}$ \\
\hline C.V.(\%) & & 22,45 & 13,57 & 14,88 & 20,64 & 18,64 \\
\hline d.m.s. & & 17,10 & 22,33 & 26,89 & 32,85 & 31,45 \\
\hline
\end{tabular}

Médias seguidas da mesma letra na coluna não diferem estatisticamente entre si, pelo teste de tukey $(\mathrm{p}>0,05)$.

** significativo a $1 \%$; significativo a $5 \%$; ${ }^{\text {ns }}$ não significativo.

${ }^{1} \operatorname{Dash}(0,5 \% \mathrm{v} / \mathrm{v}) ;{ }^{2}$ Assist $(0,5 \% \mathrm{v} / \mathrm{v})$.

${ }^{3} \mathrm{DAA}$ - dias após a aplicação.

Tabela 2 - Efeito de diferentes tratamentos químicos sobre o controle de Sida rhombifolia na cultura da mamona, em diferentes períodos após a aplicação de herbicidas. Botucatu, SP, 2009.

\begin{tabular}{|c|c|c|c|c|c|c|}
\hline \multirow{2}{*}{ Tratamentos } & Doses & \multicolumn{5}{|c|}{ (\%) Controle } \\
\hline & (g i.a./e.a. ha-1) & $3 \mathrm{DAA}^{(3)}$ & $7 \mathrm{DAA}$ & $14 \mathrm{DAA}$ & $21 \mathrm{DAA}$ & $28 \mathrm{DAA}$ \\
\hline saflufenacil $^{(1)}$ & 28 & $37,50^{\mathrm{a}}$ & $96,75 a$ & $89,00 \mathrm{a}$ & $80,50 \mathrm{ab}$ & $52,00 \mathrm{bcd}$ \\
\hline saflufenacil (1) & 40 & $37,50^{\mathrm{a}}$ & $91,25 a$ & $93,75^{\mathrm{a}}$ & $81,75 \mathrm{ab}$ & $65,00 \mathrm{abc}$ \\
\hline saflufenacil $^{(1)}$ & 56 & $40,00 \mathrm{a}$ & $96,50 a$ & $96,75^{\mathrm{a}}$ & $87,50 \mathrm{ab}$ & $87,50 \mathrm{ab}$ \\
\hline carfentrazone-ethyl ${ }^{(2)}$ & 25 & $25,00 \mathrm{a}$ & $94,50 \mathrm{a}$ & $57,50 \mathrm{~b}$ & $70,00 \mathrm{~b}$ & $45,00 \mathrm{~cd}$ \\
\hline flumioxazin $^{(2)}$ & 30 & $27,75^{\mathrm{a}}$ & $47,50 \mathrm{~b}$ & $18,75 c$ & $16,25 c$ & 20,50de \\
\hline glyphosate & 720 & ob & $24,25 c$ & $98,50^{\mathrm{a}}$ & $99,50^{\mathrm{a}}$ & $99,75 a$ \\
\hline glyphosate+saflufenacil $^{(1)}$ & $720+28$ & $27,75^{\mathrm{a}}$ & $99,00 \mathrm{a}$ & $99,00 \mathrm{a}$ & $100,00 \mathrm{a}$ & $100,00 \mathrm{a}$ \\
\hline & & $16,58^{* *}$ & $135,78^{* *}$ & $54,60 * *$ & $75,47^{* *}$ & $25,61^{* *}$ \\
\hline Fratamento & & $4,71^{*}$ & $0,09^{\text {ns }}$ & $2,36^{*}$ & $1,77^{\mathrm{ns}}$ & $2,62^{\text {ns }}$ \\
\hline Cloco $(\%)$ & & 32,29 & 9,81 & 15,49 & 13,02 & 24,66 \\
\hline d.m.s. & & 18,67 & 15,99 & 25,42 & 20,68 & 34,35 \\
\hline
\end{tabular}

Médias seguidas da mesma letra na coluna não diferem estatisticamente entre si, pelo teste de tukey $(p>0,05)$.

** significativo a $1 \%$;*significativo a $5 \%$; ${ }^{\text {ns }}$ não significativo.

${ }^{1}$ Dash $(0,5 \% \mathrm{v} / \mathrm{v}) ;{ }^{2}$ Assist $(0,5 \% \mathrm{v} / \mathrm{v})$.

${ }^{3} \mathrm{DAA}$ - dias após a aplicação.

Tabela 3 - Efeito de diferentes tratamentos químicos sobre o controle de Ipomoea grandifolia na cultura da mamona, em diferentes períodos após a aplicação de herbicidas. Botucatu, SP, 2009.

\begin{tabular}{|c|c|c|c|c|c|c|}
\hline \multirow{2}{*}{ Tratamentos } & Doses & \multicolumn{5}{|c|}{ (\%) Controle } \\
\hline & (g i.a/e.a. ha $\left.{ }^{-1}\right)$ & $3 \mathrm{DAA}^{(3)}$ & $7 \mathrm{DAA}$ & 14 DAA & $21 \mathrm{DAA}$ & $28 \mathrm{DAA}$ \\
\hline saflufenacil $^{(1)}$ & 28 & $81,25 a$ & $96,75 a$ & $98,00 \mathrm{ab}$ & $100,00 \mathrm{a}$ & $100,00 \mathrm{a}$ \\
\hline saflufenacil (1) & 40 & $92,00 \mathrm{a}$ & $98,50 \mathrm{a}$ & $99,00 \mathrm{a}$ & $100,00 a$ & $100,00 \mathrm{a}$ \\
\hline saflufenacil (1) & 56 & $88,25 a$ & $98,75 a$ & $99,50^{\mathrm{a}}$ & $99,00 \mathrm{a}$ & $100,00 \mathrm{a}$ \\
\hline carfentrazone-ethyl(2) & 25 & $83,75 a$ & $96,75 a$ & $99,25^{\mathrm{a}}$ & $100,00 \mathrm{a}$ & $99,50 \mathrm{a}$ \\
\hline flumioxazin ${ }^{(2)}$ & 30 & $58,75 a$ & $96,50 \mathrm{a}$ & $93,25 b$ & $85,75 \mathrm{a}$ & $90,00 \mathrm{ab}$ \\
\hline glyphosate & 720 & $10,50 b$ & $10,00 \mathrm{~b}$ & $5,00 \mathrm{c}$ & $13,00 \mathrm{~b}$ & $60,75 b$ \\
\hline glyphosate++ saflufenacil $^{(1)}$ & $720+28$ & $78,50 \mathrm{a}$ & $99,25 \mathrm{a}$ & $99,25^{\mathrm{a}}$ & $100,00 \mathrm{a}$ & $100,00 \mathrm{a}$ \\
\hline & & $26,64^{* *}$ & $1759,94^{* *}$ & $1822,93^{* *}$ & $147,36^{* *}$ & $21,11^{* *}$ \\
\hline $\mathrm{F}_{\text {bloco }}$ & & $0,090^{\mathrm{ns}}$ & $2,901^{\mathrm{ns}}$ & $1,769^{\mathrm{ns}}$ & $0,596^{\mathrm{ns}}$ & $0,385^{\mathrm{ns}}$ \\
\hline C.V.(\%) & & 22,81 & 2,75 & 2,80 & 9,38 & 19,02 \\
\hline d.m.s. & & 33,34 & 4,86 & 4,92 & 16,62 & 36,69 \\
\hline
\end{tabular}

Médias seguidas da mesma letra na coluna não diferem estatisticamente entre si, pelo teste de tukey (p >0,05).

** significativo a $1 \%$;*significativo a $5 \%$; ${ }^{\text {ns }}$ não significativo.

${ }^{1}$ Dash $(0,5 \% \mathrm{v} / \mathrm{v}) ;{ }^{2}$ Assist $(0,5 \% \mathrm{v} / \mathrm{v})$.

${ }^{3} \mathrm{DAA}$ - dias após a aplicação. 
Aos 21 DAA dos herbicidas, observa-se que apenas os usos deflumioxazin, glyphosate glyphosate + saflufenacil proporcionaram controles considerados debomà excelente da planta daninha de poaia-branca. Ao final das avaliações, $28 \mathrm{DAA}$, registraram-se que as duas doses maiores de saflufenacil, flumioxazin, glyphosate e glyphosate + saflufenacil controlaram as plantas de poaia-branca com notas que variaram de bom a excelente. MAcIEL et al. (2008) observaram controle satisfatório de poaia-branca, onde as notas encontravam-se $\leq 80 \%$.

Para S. rhombifolia (guaxuma), nota-se que aos 3 DAA dos herbicidas que nenhum dos tratamentos testados mostraram-se satisfatórios no controle da planta daninha (Tabela 2). Já aos 7 DAA, registrase um excelente controle das plantas daninhas de guaxuma, exceção dos herbicidas flumioxazin e glyphosate aplicado de forma isolada.

Aos 14 DAA, ocorreu um aumento do controle dos tratamentos com glyphosate que para a mistura já havia ocorrido no $7^{\circ}$ DAA. Não houve diferença significativa das doses de saflufenacil para a mistura do glyphosate, o que demonstra um controle satisfatório desta planta daninha pela molécula de saflufenacil. O efeito do herbicida flumioxazin, neste período, sobre plantas de $S$. rhombifolia, não obteve controle satisfatório, na qual se manteve até os 28 DAA.

Observa-se aos 3 DAA dos herbicidas que o controle das plantas de I. grandifolia (corda-de-viola) com os herbicidas saflufenacil nas suas diferentes doses testadas e carfentrazone-ethyl foram superiores a $80 \%$ (Tabela 3). Segundo MonQuero et al. (2001), as plantas de I. grandifolia foram controladas com $97 \%$ com a aplicação de carfentrazone-ethyl isoladamente, demonstrando um excelente controle desta. Os herbicidas flumioxazin e glyphosate aplicado de forma isolada apresentaram-se ineficientes no controle da planta daninha. Já a aplicação da mistura de glyphosate+saflufenacil demonstrou um controle apenas satisfatório.

Aos 7DAA dos herbicidas registrou-se um elevado incremento no controle em todos os tratamentos químicos testados, exceção a aplicação do glyphosate isoladamente. Já aos 14 DAA, nota-se, ainda, que apenas a aplicação isolada de glyphosate manteve-se ineficiente no controle das plantas de corda-de-viola. Tal fato não corrobora os resultados obtidos por DiAs etal. (2007), que observaram um controle eficiente da planta daninha utilizando a mesma dose ora testada e período avaliado (14 DAA).

Aos 21 e 28 DAA dos herbicidas os resultados foram semelhantes, sendo que todos os herbicidas e doses testadas foram eficientes no controle das plantas de I. grandifolia, exceção apenas da aplicação isolada do glyphosate. Também PROcóPIO (2007) observou que o glyphosate apresentava-se ineficiente no controle da planta daninha. Em outro estudo, LACERDA; Victoria FilHo (2004) verificaram um controle insatisfatório desta espécie com uso de glyphosate, como ora observados.

Um aspecto importante a ressaltar quanto ao herbicida saflufenacil, que apresentou um excelente controle das plantas de I. grandifolia mesmo em doses muito baixas, foi que DiAs et al. (2007) observaram excelentes resultados como foram mostrados anteriormente.

O saflufenacil foi eficiente para todas as plantas daninhas estudadas. Na dose maior $\left(56 \mathrm{~g} \mathrm{ha}^{-1}\right)$ obteve controle satisfatório (superior a $80 \%$ ) para todas as plantas daninhas. Os tratamentos com menor eficiência nos controles são carfentrazone-ethyl seguido de flumioxazin. O herbicida carfentrazone-ethyl foi eficaz no controle de I. grandifolia, porém para $R$. brasiliensis e S. rhombifolia os controles não foram satisfatórios. Otratamento com flumioxazin foi o que obteve menor controle em S. rhombifolia, controlando bem as plantas de R. brasiliensis e I. grandifolia.

A mistura de glyphosate com saflufenacil e glyphosate foi o que manteve os resultados mais expressivos, controlando todas as plantas daninhas estudadas. A aplicação com glyphosatenão controlou I. grandifolia ao nível de controle aceitável.

\section{REFERÊNCIAS}

AZEVEDO, D.M.P.; SEVERINO, L.S. Cultivo da mamona: plantas daninhas. 2.ed., 2006. (EMBRAPA ALGODÃO, n. 4). Disponível em: <http:/ / www.sistemasdeproducao.cnptia.embrapa.br/FontesHTML/Mamona/ CultivodaMamona_2ed/plantasdaninhas.html>. Acesso em: 3 nov. 2011.

DIAS, A.C.R.; YOTSUMOTO, T.; CARDINALI, V.C.B.; NICOLAI, M.; CHRISTOFFOLETI, P.J. Eficiência agronômica do herbicida glyphosate isolado e em mistura com outros herbicidas no controle de quatro espécies de corda de viola. In: SIMPÓSIO INTERNACIONAL SOBRE GLYPHOSATE, 1., 2007, Botucatu. Trabalhos Científicos. Botucatu: Aldeia Norte, 2007. p.23-26.

DUKE, S.O.; LYDON, J.; BECERRIL, J.M.; SHERMAN, T.D.; LEHNEN JUNIOR, L.P.; MATSUMOTO, H. Protoporphyrinogen oxidase-inhibiting herbicides. Weed Science., v.39, p.465-473, 1991.

EMBRAPA, Zoneamento da Mamona no Nordeste, Disponível em; <http:/ / www.cnpa.embrapa.br/produtos / mamona/zoneamento.html>. Acesso em: 3 nov. 2011.

GROSSMANN, K.; NIGGEWEG, R.; CHRISTIANSEN, N.; LOOSER, R.; EHRHARDT, T. The Herbicide Saflufenacil (KixorTM) is a New Inhibitor of Protoporphyrinogen IX Oxidase Activity. Weed Science, v.58, n.1, p.1-9, 2010. 
LACERDA, A.L.S.; VICTORIA FILHO, R. Eficácia de Roundup Ready em dois estádios fenológicos de desenvolvimento de Commelina benghalensis e Ipomoea grandifolia. Boletim Informativo SBCPD, v.10, p 223, 2004. Suplemento. Trabalho apresentado no CONGRESSO BRASILEIRO DA CIÊNCIA DAS PLANTAS DANINHAS 24., 2004, São Paedro, SP.

MACIEL, C.D.G.; POLETINE, J.P.; VELINI, E.D.; AMARAL, J.G.C.; ZANI, L.P.; SANTOS, R.F.; RODRIGUES, M.; RAIMONDI, M.A.; RIBEIRO, R.B. Possibilidade de aplicação de misturas de herbicidas de ação total com jato dirigido em mamoneira de porte anão. Planta Daninha, v.26, n.2, p. 457-464, 2008.

MACIEL, C.D.G.; GAVA, F.; VELINI, E.D.; POLETINE, J.P.; AMARAL, J.G.C.; MARTINS, F.M. Períodos de interferência de plantas daninhas na cultura da mamona - Cultivar AL Guarany 2002. In: CONGRESSO BRASILEIRO DA CIÊNCIA DAS PLANTAS DANINHAS, 24. 2004. São Pedro. Anais. Londrina: SBCPD/Sociedade Brasileira da Ciência das Plantas Daninhas, 2004. 1 CD-ROM.
MONQUERO, P.A.; CHRISTOFFOLETI, P.J.; SANTOS, C.T.D. Glyphosate em mistura com herbicidas alternativos para o manejo de plantas daninhas. Planta Daninha, v.19, n.3, p.375-380, 2001.

PROCÓPIO, S.O.; MENEZES, C.C.E.; BETTA, L.; BETTA, M. Utilização de chorumuron-ethyl e imazathapyr na cultura da Soja Roundup Ready. Planta Daninha, v.25, n.2, p.365-373, 2007.

SHANER, D.; BRIDGES, D. Inhibitors of aromatic amino acid biosyntesis (glyphosate). In: SHANER, D.; BRIDGES, D. (Ed.). Herbicide action course. West Lafayette: Purdue University, 2003. p.514-529.

SOCIEDADE BRASILEIRA DA CIÊNCIA DAS PLANTAS DANINHAS. Procedimentos para instalação, avaliação e análise de experimentos com herbicidas. Londrina: SBCPD, 1995. 42p.

Recebido em 12/7/10

Aceito em 3/11/11 